\title{
Hommage à Pierre-Gilles de Gennes
}

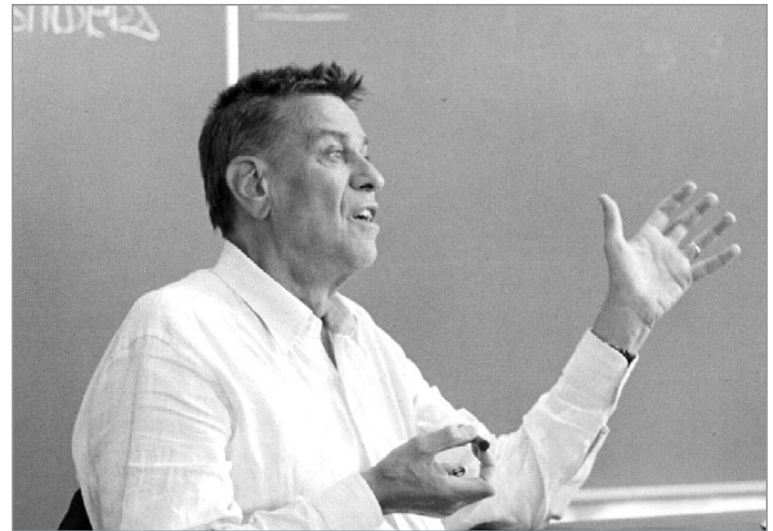

Pierre-Gilles de Gennes nous a quittés : c'est un très grand physicien qui disparaît, et avec lui une façon unique d'aborder la science. Tous ceux qui l'ont approché resteront marqués par son style si personnel, fait d'une grande élégance appuyée sur des connaissances très profondes. Son attention était sans cesse en éveil, son imagination toujours prête à s'enthousiasmer pour une nouvelle idée. Son charisme était exceptionnel : il a marqué toute une génération de physiciens de la matière condensée. Jusqu'à ses derniers jours il travaillait encore, bien que très affaibli, à ces problèmes relatifs au fonctionnement de la mémoire qu'il avait évoqués pour l'ouverture de l'Année Mondiale de la Physique en 2005, devant une assemblée de lycéens fascinés.

Les sujets de recherche auxquels il a apporté une contribution majeure feront l'objet, dans un prochain numéro de Reflets de la physique, d'articles rédigés par ses principaux collaborateurs. Ils évoqueront ses travaux sur les cristaux liquides, les polymères et les colloïdes, qui lui ont valu d'être considéré comme le père de la physique de la matière molle et de recevoir le prix Nobel en 1991. D’autres articles traiteront de la supraconductivité, un problème auquel il a travaillé tout au long de sa carrière, et de la physique des milieux granulaires qui est toujours aussi riche. Ses collaborateurs les plus récents retraceront son parcours en biophysique, un domaine qu'il a introduit dans cette École de Physique et Chimie de Paris qu'il a longtemps dirigée avec tant de succès. Bien d'autres témoignages viendront illustrer sa curiosité d'esprit pour une multitude de problèmes tirés de la vie quotidienne, auxquels il semblait s'amuser à apporter des explications simples. II aimait qu'on lui pose des questions et ne craignait pas de se tromper parfois dans ses réponses : la fécondité de l'erreur en science avait d'ailleurs constitué un thème central de sa leçon inaugurale au Collège de France, ce dont je me souviens, à l'occasion, comme d'une idée forte.

Je voudrais rappeler ici combien Pierre-Gilles de Gennes aimait partager ce qu'il avait compris. Ses cours étaient brillants et très suivis, tant au Collège de France qu'à l'École des Houches où il faisait de fréquents séjours, accompagné d'une nombreuse famille. On ne peut compter le nombre de ses interventions dans les lycées et les collèges dans l'année qui a suivi son prix Nobel, suscitant, n'en doutons pas, un nombre considérable de vocations pour la science. Et lorsqu'il fut en 1982 président de la SFP, il insistait déjà sur l'importance des liens entre notre société savante et les enseignants du secondaire. La SFP a aujourd'hui passé commande à leur intention d'un ouvrage accessible, décrivant ses contributions majeures à la physique actuelle.

Il est intéressant de relire les deux éditoriaux écrits par Pierre-Gilles de Gennes pour le Bulletin de la SFP. II y dénonçait le recrutement presque exclusif dans le monde académique, déplorant la coupure avec celui des ingénieurs. II prévoyait l'étiolement à terme d'une société de physique, si elle devait se couper de ses applications et se désintéresser des problèmes de société qui lui sont liés. II appelait de ses vœux la réunion de l'ensemble des sociétés représentant les sciences physiques : ce message si lucide mérite aujourd'hui d'être rappelé.

Ainsi I'héritage de Pierre-Gilles de Gennes est immense. Il va continuer à nous inspirer pendant longtemps. 